\title{
Acute aortic insufficiency in a patient with presumed Reiter's syndrome*
}

Patricia Misukiewicz, Richard W Carlson, Laurie Rowan, Neil Levitt, Cheryl Rudnick, Tusar Desai

\begin{abstract}
Cardiac disease is rare in patients with Reiter's syndrome. There have been 15 reported cases of aortic insufficiency in patients with Reiter's syndrome, with the aortic insufficiency developing over several years. This paper reports the case of a black HLA-B27 negative woman who presented with Reiter's syndrome and acute aortic insufficiency. An antecedent streptococcal infection is suggested as the inciting factor. To our knowledge, this is the first report of Reiter's syndrome in a black woman with acute aortic insufficiency.
\end{abstract}

\section{Case report}

A 42 year old black woman presented with an eight day history of bilateral photophobia, reddened sclera, sore throat, non-bilious vomiting, diarrhoea, a painful left knee, urinary frequency and urgency, fever, shaking chills, and neck stiffness. The patient had a previous history of scleritis, with recurrent episodes over a period of eight years. She did not have any previous history of sexually transmited disease, and her last sexual intercourse was more than a year earlier. Hypertension had been diagnosed three months before her hospital admission and was well controlled with a thiazide diuretic. There was no relevant family history or seronegative spondyloarthropathy.

The patient was febrile and the sclerae were markedly injected; an ophthalmological examination showed anterior uveitis. Although her tonsils were enlarged with exudates, no mucocutaneous lesions were noted. Neck pain and stiffness on flexion were observed. Lung, cardiovascular, abdominal, pelvic, and neurological examinations were normal. The left knee was tender and warm but the range of motion was full and there was no effusion. The remainder of the musculoskeletal examination was normal except for local tenderness of the lumbar spine. Lumbar puncture showed normal pressure and chemistry; urine analysis showed 10-20 white blood cells/high power field and no red blood cells, casts, nor proteinuria. Other findings included a white blood cell count of $10.6 \times 10^{9} / 1$ and an increased erythrocyte sedimentation rate of $56 \mathrm{~mm} /$ hour. Radiographs of the chest, left knee, and sacroiliac joints were normal. An electrocardiograph showed normal sinus rhythm without evidence of any conduction abnormality.

The patient was treated empirically with intravenous gentamicin. She remained febrile, although cultures of blood, urine, rectal, and cervical swabs obtained before treatment with antibiotics were all negative. The titre of antibodies to streptolysin was 800 Todd units and the streptozyme titre was $1 / 600$. A throat culture was positive for group $\mathrm{G}$ streptococcus. Rheumatoid factor was positive but only in $1 / 20$ dilution. $C$ reactive protein was $120 \mathrm{mg} / \mathrm{l}(0-0.8$ normal value).

On the third day after admission she developed respiratory distress, tachypnoea, tachycardia, and hypotension (arterial pressure 90/68 $\mathrm{mmHg})$. A pericardial rub and an aortic insufficiency murmur developed. Acute pulmonary oedema followed and the patient was intubated and placed on mechanical ventilation (fig 1). A chest computed tomography scan showed a large ascending aorta $(4 \mathrm{~cm})$ and dilatation of the aortic root, bilateral pleural effusions, pulmonary vascular congestion, and mediastinal
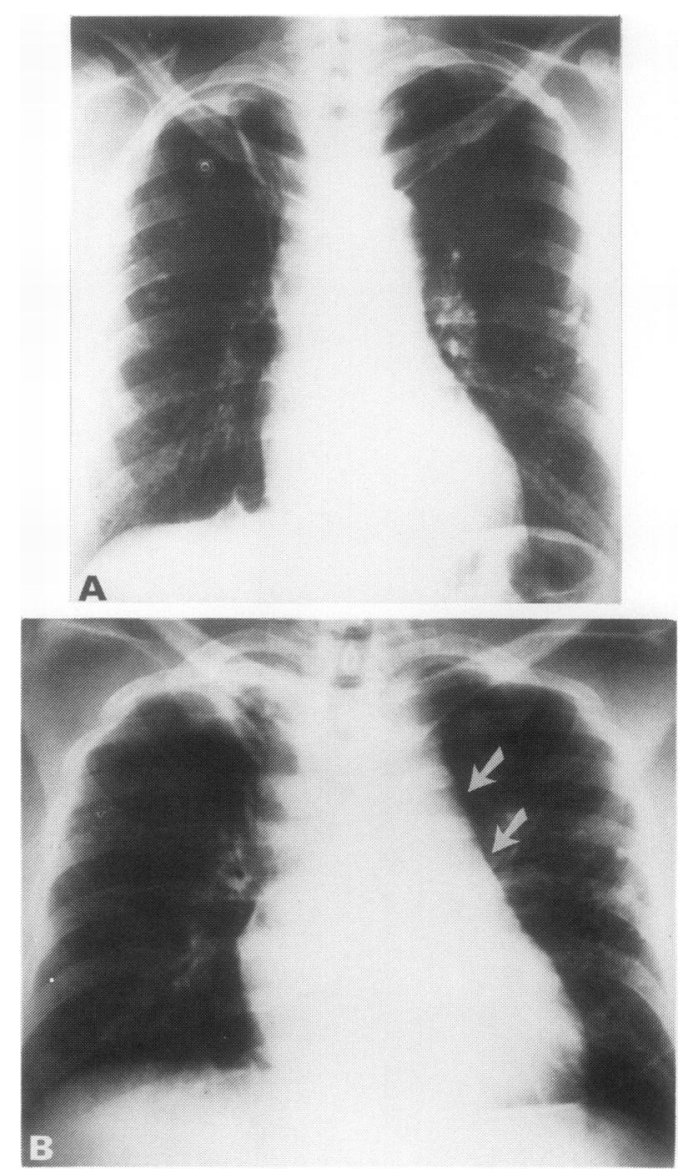

Figure 1 Chest radiographs $(A)$ on admission showing no acute pulmonary infiltrate nor cardiac enlargementcalcification is seen owing to a history of tuberculosis-and $(B)$ on the third day after admission showing loss of the aortic arch, a widened mediastinum, and venous engorgement (arrows). 


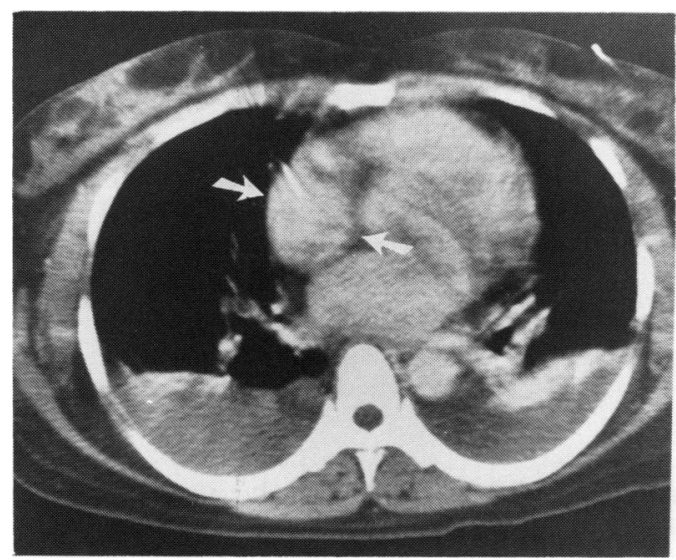

Figure 2 Chest computed tomograph showing a large $(4 \mathrm{~cm})$ ascending aorta (arrows).

fluid (fig 2). Moderate aortic insufficiency with thickened mitral leaflets was observed on the echocardiogram. No vegetations of the aortic valve were found. The haemodynamic profile was consistent with acute aortic insufficiency with cardiac decompensation (cardiac output $5 \cdot 7 \mathrm{l} / \mathrm{min}$, central venous pressure 22 , pulmonary capillary wedge 33 ; heart rate $115 /$ minute). The patient was stabilised with intravenous nitroprusside, furosemide, and nitroglycerin. The following day a $23 \mathrm{~mm}$ St Jude prosthetic aortic valve was inserted. The operation showed diffuse aortitis with annular ectasia. There were no annular abscesses or vegetations. A biopsy of the aortic valve showed myxomatous degeneration with no inflammatory cellular infiltrate. Her haemodynamic status stabilised within 48 hours but recovery after the operation was complicated by a low grade fever. No infectious aetiology was discovered. The patient was discharged 21 days after the operation.

One month later she presented to the emergency room with syncope and shock. Within minutes she developed bradycardia and cardiopulmonary arrest. Resuscitation was unsuccessful. Necropsy showed no gross abnormalities of the heart, pericardium, aorta, or prosthetic valve. Although the haemoglobin was $89 \mathrm{~g} / \mathrm{l}$ and the prothrombin time was minimally prolonged, there was no sign of massive bleeding at necropsy. Mechanical failure of the valve was suspected as the cause of death.

\section{Discussion}

Reiter's syndrome is a clinical diagnosis based on several findings. ${ }^{1}$ This patient fits the clinical criteria proposed by the American Rheumatism Association $^{2}$ for Reiter's syndrome: a reactive arthritis which follows a cystitis/urethritis or dysentery and is associated with an anterior uveitis. Reiter's syndrome usually follows an infection with chlamydia, shigella, salmonella, campylobacter, or yersinia. There is an increased risk of Reiter's syndrome in HLA-B27 positive patients following infection with these organisms. ${ }^{3}$ Although $80-90 \%$ of patients with Reiter's syndrome have the HLA-B27 gene, this association is weak in black patients ${ }^{4}$; this patient was HLA-B27 negative.

In this patient the inciting agent was not obvious; stool cultures and tests for the chlamydia antibody were negative. The development of Reiter's syndrome following a streptococcal infection is rare. Thirteen cases of this association have been reported. ${ }^{5}$ Antistreptolysin $\mathrm{O}$ and streptozyme titres were positive in this patient and we therefore speculate that an antecedent streptococcal infection was the causative agent.

The course of this patient was fulminant; she rapidly developed acute aortic insufficiency and pericarditis. Cardiac disease in Reiter's syndrome is rare, but has been noted. Neu et al reported myocarditis and pericarditis in 45 of 364 patients with electrocardiographic changes. ${ }^{6}$ Aortic insufficiency has been described in only 15 patients with Reiter's syndrome. ${ }^{7}$ Aortic insufficiency developed an average of 15 years after the onset of the syndrome, however, and was associated with aortic valve conduction abnormalities in most instances. In contrast with Reiter's syndrome, acute aortic insufficiency has been reported in patients with ankylosing spondylitis, ${ }^{8}$ and the pathology of the aortic valve in one patient showed myxomatous changes as in this case. ${ }^{9}$ The patient described here developed acute severe aortic insufficiency and pericarditis with haemodynamic instability over three days. Findings included increased pulmonary artery occlusive pressure, right atrial hypertension, and a reduction of stroke volume related to the inability of the acutely failing ventricle to dilate. Acute pulmonary oedema supervened and urgent replacement of the aortic valve was required.

This patient is unique for several reasons. The patient was a black woman without the HLA-B27 gene who presented clinically as having Reiter's syndrome (or reactive arthritis) associated with a purulent pharyngitis. The increased antistreptolysin $\mathrm{O}$ and streptozyme titres suggest that streptococcus (group G) was the inciting agent. $\beta$ Haemolytic, non-group A streptococcus has been suggested to cause reactive arthritis, ${ }^{10}$ but this has not been reported definitively. The rapid development of aortic insufficiency has not been reported previously in patients with Reiter's syndrome.

The authors express their appreciation to Janet Misukiewicz for her expert typing skills in the preparation of this manuscript.

1 Good A E. Reiter's disease: a review with special attention to cardiovascular and neurologic sequelae. Semin Arthritis Rheum 1974; 3: 253-86.

2 Wilkens R D, Arnett F C, Bitter T, et al. Reiter's syndrome: evaluation of preliminary criteria for definitive disease. Arthritis Rheum 1981; 24: 844-9.

3 Schumacher H R, ed. Primer on rheumatic diseases. Vol VI. Richmond. 1988: 147-50.

4 Khan M A, Askari A D, Braun W E, Aponte C J. Low association of HLA-B27 with Reiter's syndrome in blacks. Ann Intern Med 1979; 90: 202-3.

5 Olhagen B. Chronic uropolyarthritis in males. Acta Med Scand 1960; 168: 339-45.

6 Neu L T, Reider R A, Mack R E. Cardiac involvement in Reiter's disease: report of a case with review of the literature. Ann Intern Med 1960; 53: 215-9.

7 Paulus $\mathrm{H}$ E, Pearson C M, Pitts W. Aortic insufficiency in five patients with Reiter's syndrome. $A m \mathcal{F}$ Med 1972; 53: 464-72.

8 Stewart S R, Robbins D L, Castles J J. Acute fulminant aortic and mitral insufficiency in ankylosing spondylitis. N Engl F Med 1978; 299: 1048.

9 Demoulin J C, Lespagnard J, Bertholet M, Soumagne D. Acute fulminant regurgitation in ankylosing spondylitis. Am Heart $\mathcal{f}$ 1983; 105: 359-61.

10 Valtonen V, Leirisalo M, Pentikaninen P, et al. Triggering infections in reactive arthritis. Scand F Rheumatol 1985; 44: 399-405. 